\title{
BeppoSAX observations of Mrk 841 and Mrk 335
}

\author{
S. Bianchi ${ }^{1}$, G. Matt ${ }^{1}$, F. Haardt ${ }^{2}$, L. Maraschi ${ }^{3}$, F. Nicastro ${ }^{4}$, G. C. Perola ${ }^{1}$, P. O. Petrucci ${ }^{3}$, and L. Piro ${ }^{5}$ \\ 1 Dipartimento di Fisica, Università degli Studi "Roma Tre", Via della Vasca Navale 84, 00146 Roma, Italy \\ 2 Dipartimento di Scienze, Università dell'Insubria, Via Lucini 3, 22100 Como, Italy \\ 3 Osservatorio Astronomico di Brera, Via Brera 28, 20121 Milano, Italy \\ 4 Harvard-Smithsonian Center for Astrophysics, 60 Garden st., Cambridge, MA 02138, USA \\ ${ }^{5}$ Istituto di Astrofisica Spaziale, C.N.R., Via Fosso del Cavaliere, 00133 Roma, Italy
}

Received 30 January 2001 / Accepted 4 July 2001

\begin{abstract}
We present and discuss BeppoSAX observations of Mrk 841 and Mrk 335, two Seyfert 1 galaxies in which previous observations have established the presence of soft excesses. We confirm the soft excess in both sources, even if for Mrk 841 a warm absorber provides a fit almost as good as the one with a true excess. As far as the hard X-ray continuum is concerned, a Comptonization model provides a fit as good as a power law and a physically sound solution for Mrk 841. For Mrk 335, the Comptonization model gives a result which is somewhat better on statistical ground, but rather problematic on physical ground. The most interesting results regard the reprocessing components. For Mrk 841 we find a very large reflection continuum but an almost normal iron line equivalent width even if, within the errors, a solution in which both components are a factor $\sim 2$ larger than expected for an accretion disc is still marginally acceptable. If this is the case, an anisotropy of the primary emission seems the best explanation. On the contrary, in Mrk 335 we find a very large iron line $E W$ but a reflection component not accordingly large. In this case, the best solution seems to be in terms of reflection from an ionized disc.
\end{abstract}

Key words. galaxies: individual: Mrk 841, Mrk 335 - galaxies: Seyfert - X-rays: galaxies

\section{Introduction}

Soft excesses, i.e. emission below $\sim 1 \mathrm{keV}$ in excess of the power law component dominating the X-ray spectrum above that energy, were discovered in Seyfert 1 galaxies by EXOSAT (e.g. Arnaud et al. 1985; Turner \& Pounds 1989) and later confirmed by ROSAT (e.g. Walter \& Fink 1993; Walter et al. 1994; Piro et al. 1997). In the first case, however, it is possible that the excesses were somewhat overestimated because the Compton reflection component had not yet been discovered at that time, and the best-fit hard X-ray spectra were therefore found flatter than real. In the second case, the limited ROSAT bandwidth implied that data from another satellite (usually either GINGA or ASCA), not always simultaneous, had to be employed, with related uncertainties. Moreover, it is possible that most of the published ROSAT power law indices are steeper than real due to calibration problems (Fiore et al. 1994; Iwasawa et al. 1999; Mineo et al. 2000), again overestimating (if not creating altogether) soft excesses, when compared with higher energy spectra. ASCA energy band goes down only to $0.6 \mathrm{keV}$ (and only with

Send offprint requests to: S. Bianchi,

e-mail: bianchi@fis.uniroma3.it the SIS instruments), not enough to study the majority of soft excesses in Seyfert 1s, which were found to be relevant below that energy.

Despite the lower sensitivity with respect to both ROSAT and ASCA in the overlapping energy ranges, BeppoSAX (Boella et al. 1997), thanks to the broad energy covering (0.1-200 keV), may overcome some of the problems affecting previous studies on soft excesses. While confirming the commonness of soft excesses in both Narrow Line Seyfert 1 galaxies (Comastri et al. 2000, and references therein) and in high luminosity PG quasars (Mineo et al. 2000), up to now BeppoSAX observations seem indicate that soft excesses are the exception rather than the rule in classical, broad line Seyfert 1 galaxies (Matt 2000 and references therein). We therefore decided to study with BeppoSAX two Seyfert 1s in which the claim of the presence of soft excesses have been among the strongest in the past.

Mrk 841 is indeed the first source in which the soft excess was discovered (Arnaud et al. 1985). Since then, Mrk 841 has been extensively studied, remaining one of the best case for this component (George et al. 1993; Nandra et al. 1995). In particular, it is one of the three (out of 24) sources in the ASCA sample of Reynolds (1997) 
in which "soft excess is clearly seen in the data" (the other two sources being Mrk 335, see below, and NGC 4051). The source is interesting also in other respects: the intrinsic power law index varies strongly between different observations (which, by the way, may confuse the soft excess estimate if non-simultaneous observations in different bands are compared); a fairly strong fluorescent iron $\mathrm{K} \alpha$ line $(E W \sim 450 \mathrm{eV})$ was detected by GINGA (George et al. 1993); in one GINGA observation the source was apparently reflection-dominated (George et al. 1993), similarly to what happened recently to NGC 4051 (Guainazzi et al. 1998). All these facts make Mrk 841 a source worth investigating even independently of the soft excess issue.

Mrk 335 is another source in the Reynolds (1997) sample in which the soft excess is clearly present, confirming previous findings by EXOSAT (Turner \& Pounds 1989) and BBXRT (Turner et al. 1993a). Similarly to Mrk 841, no clear evidence for warm absorption is present in ASCA data (Reynolds 1997; George et al. 1998), but Turner et al. (1993b) previously found evidence for a warm absorber in the ROSAT data. Evidence for such an absorber was also found by Orr (2000) in the same BeppoSAX data we are discussing in this paper. Both Reynolds (1997) and Orr (2000) detected a strong iron $\mathrm{K} \alpha$ line, with $E W \sim 560$ and $230 \mathrm{eV}$, respectively. It should be noted that Mrk 335 is often classified as a NLSy1, but has a relatively high $\mathrm{H} \beta$ FWHM (1640 $\mathrm{kms}^{-1}$, Wang et al. 1996, close to the conventional boundary for this class of objects of $2000 \mathrm{~km} \mathrm{~s}^{-1}$ ), and a rather normal power law index (see also below), and may therefore be considered a borderline object.

\section{Observations and data reduction}

For both sources, the observing dates, exposure times, count rates (for the LECS, MECS and the PDS instruments, the latter referring to two units, as at any time only half detector is on source), fluxes and luminosities are summarized in Table 1. While the Mrk 841 data are presented here for the first time, results from the BeppoSAX observation of Mrk 335 have already been presented, but not discussed in detail, by Orr (2000). For the imaging instruments, light curves and spectra have been extracted from $4^{\prime}$ (LECS) and $3^{\prime}$ (MECS) circles centred on the sources. Background spectra taken in the same regions from blank sky observations have been used for subtraction. Regarding the PDS, background subtracted spectra automatically generated at the BeppoSAX Scientific Data Centre (SDC) have been used. Variable Rise Time thresholds have been adopted.

\section{Temporal analysis}

\subsection{Mrk 841}

The LECS (0.1-4 keV), MECS (1.8-10.5 keV) and PDS (13-100 keV) light curves of Mrk 841 are shown in Fig. 1. The source varies up to $50 \%$ on time-scales of

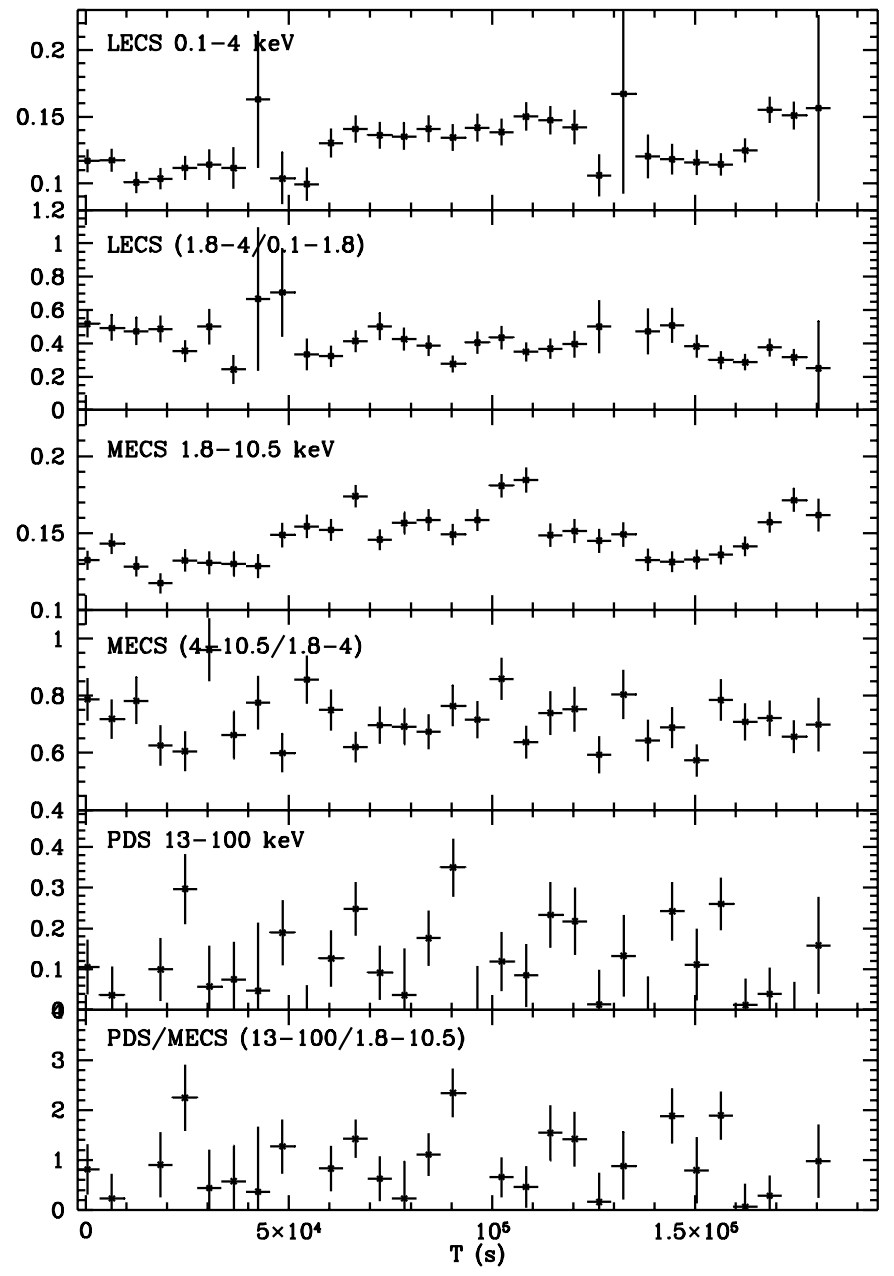

Fig. 1. Mrk 841. From top to bottom: LECS light curve and hardness ratio; MECS light curve and hardness ratio; PDS light curve (background subtracted) and PDS/MECS hardness ratio.

tens of thousand seconds. To search for spectral variability, we have also plotted the $(1.8-4) /(0.1-1.8)$ LECS, the $(4-10.5) /(1.8-4)$ MECS and the PDS/MECS hardness ratios. There is marginal evidence for moderate and rather erratic spectral variability, not correlated with flux variations.

\subsection{Mrk 335}

A similar temporal analysis has been made for Mrk 335 (see Fig. 2). During the observation the source varied up to a factor $\sim 2$ in both the LECS and MECS. There is marginal evidence for spectral variability, but also in this case not correlated with flux variations.

\section{Spectral analysis}

For both sources, the spectra have been averaged over the entire observations, as spectral variability is modest. As usual, in the fits the normalization of the LECS and PDS with respect to MECS has been kept free and fixed to 0.8 , respectively. In the following, all quoted errors correspond 
Table 1. Observation epochs and mean count rates, fluxes and luminosities $\left(H_{0}=50 \mathrm{~km} \mathrm{~s}^{-1} \mathrm{Mpc}^{-1}\right)$.

\begin{tabular}{|c|c|c|c|c|c|c|c|c|c|}
\hline \multirow[t]{2}{*}{ Target } & \multirow[t]{2}{*}{ Start Date } & \multicolumn{2}{|c|}{$\begin{array}{c}\text { LECS } \\
(0.1-4 \mathrm{keV})\end{array}$} & \multicolumn{2}{|c|}{$\begin{array}{c}\text { MECS } \\
(1.8-10.5 \mathrm{keV})\end{array}$} & \multicolumn{2}{|c|}{$\begin{array}{c}\text { PDS } \\
(13-200 \mathrm{keV})\end{array}$} & \multirow{2}{*}{$\begin{array}{c}\text { Flux } \\
(2-10 \mathrm{keV}) \\
\text { erg } \mathrm{cm}^{-2} \mathrm{~s}^{-1}\end{array}$} & \multirow{2}{*}{$\begin{array}{c}\text { Lum. } \\
(2-10 \mathrm{keV}) \\
\mathrm{erg} \mathrm{s}^{-1}\end{array}$} \\
\hline & & $\begin{array}{l}t_{\exp } \\
(\mathrm{s})\end{array}$ & $\begin{array}{c}C R \\
\left(\operatorname{cts~s}^{-1}\right)\end{array}$ & $\begin{array}{l}t_{\exp } \\
(\mathrm{s})\end{array}$ & $\begin{array}{c}C R \\
\left(\operatorname{cts~s}^{-1}\right)\end{array}$ & $\begin{array}{l}t_{\exp } \\
(\mathrm{s})\end{array}$ & $\begin{array}{c}C R \\
\left(\operatorname{cts~s}^{-1}\right)\end{array}$ & & \\
\hline Mrk 841 & $\begin{array}{c}\text { 1999-Jul.-30 } \\
(12 \mathrm{~h} 15 \mathrm{~m} 49 \mathrm{~s} \text { UT })\end{array}$ & 32760 & $\begin{array}{c}0.124 \\
\pm 0.002\end{array}$ & 87621 & $\begin{array}{c}0.141 \\
\pm 0.001\end{array}$ & 83008 & $\begin{array}{c}0.12 \\
\pm 0.02\end{array}$ & $1.3 \times 10^{-11}$ & $7.3 \times 10^{43}$ \\
\hline Mrk 335 & $\begin{array}{c}\text { 1998-Dec.-10 } \\
(07 \mathrm{~h} 46 \mathrm{~m} 28 \mathrm{~s} \text { UT })\end{array}$ & 40544 & $\begin{array}{c}0.076 \\
\pm 0.001\end{array}$ & 86455 & $\begin{array}{c}0.091 \\
\pm 0.001\end{array}$ & 85178 & $\begin{array}{c}0.05 \\
\pm 0.02\end{array}$ & $0.8 \times 10^{-11}$ & $2.3 \times 10^{43}$ \\
\hline
\end{tabular}

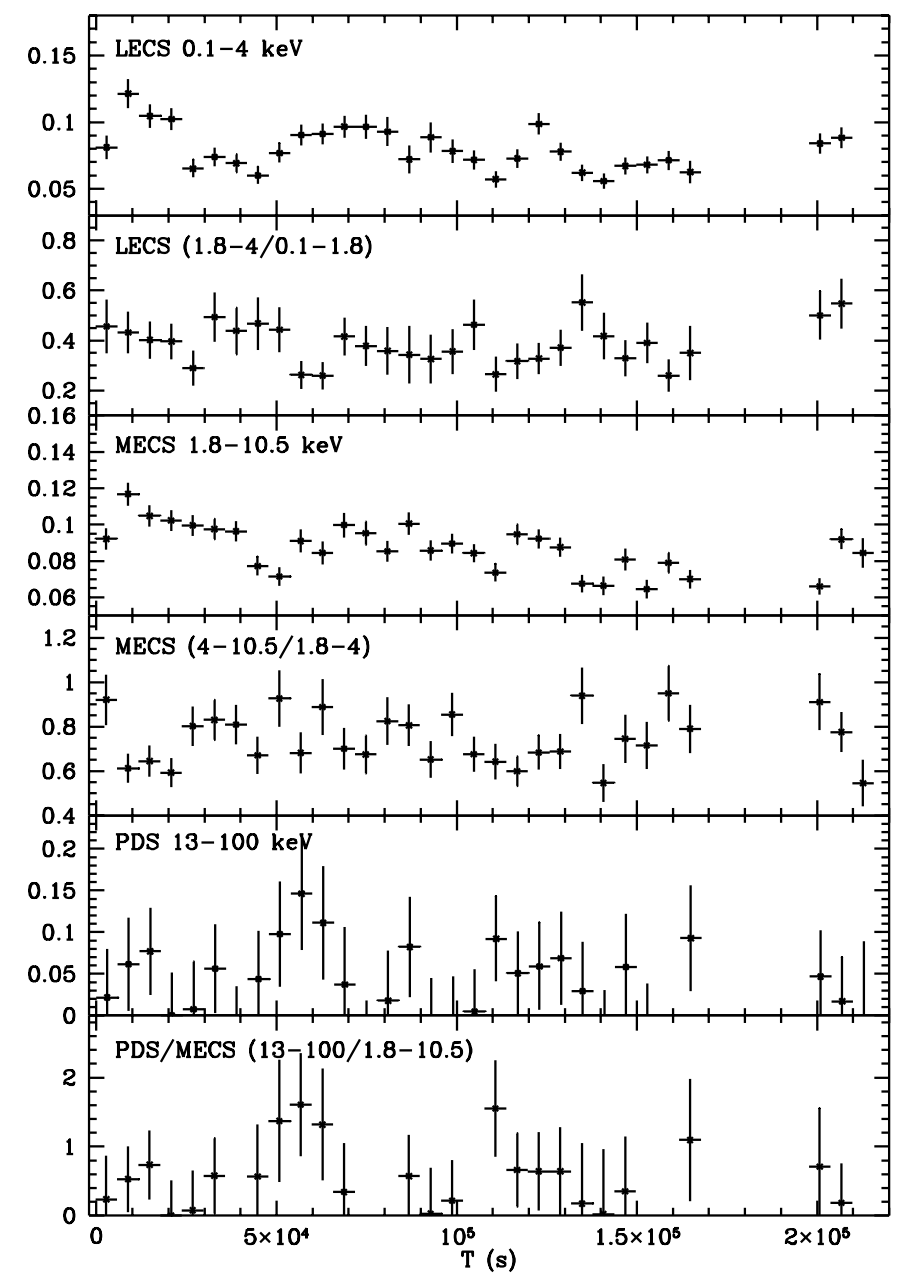

Fig. 2. Mrk 335. From top to bottom: LECS light curve and hardness ratio; MECS light curve and hardness ratio; PDS light curve (background subtracted) and PDS/MECS hardness ratio.

to the 90 percent confidence level for one interesting parameter $\left(\Delta \chi^{2}=2.7\right)$. All spectal fits were performed with the XsPEC package (version 10).

\subsection{Mrk 841}

The data from the three instruments were fitted together. We first fitted the spectra above $2 \mathrm{keV}$ with a model composed by a power law with exponential cut-off absorbed by the Galactic $N_{\mathrm{H}}\left(2.34 \times 10^{20} \mathrm{~cm}^{-2}\right.$, obtained using the

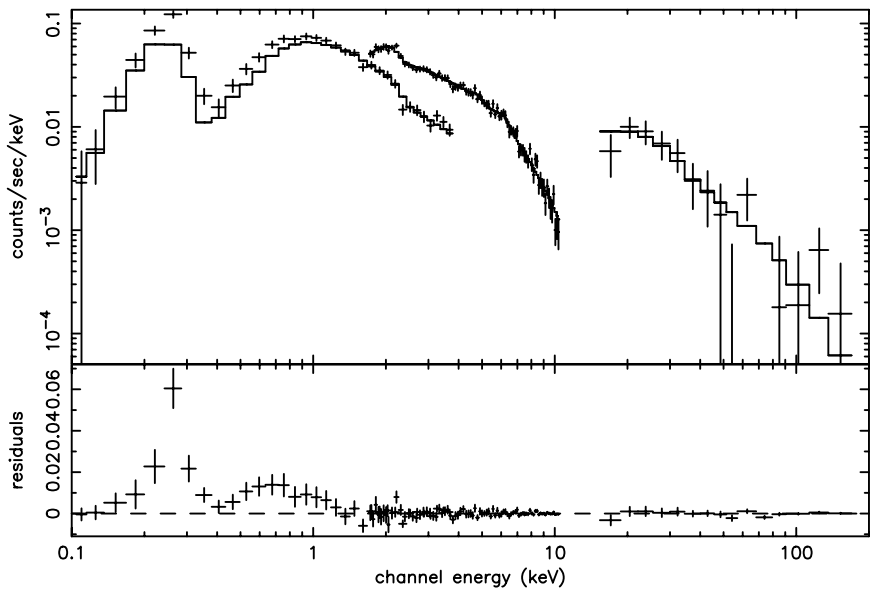

Fig. 3. BeppoSAX LECS+MECS+PDS data and best fit model above $2 \mathrm{keV}$ for Mrk 841, then extrapolated to lower energies to illustrate the presence of a soft excess.

W3NH HEASARC tool), plus a reflection component and an iron $\mathrm{K} \alpha$ line. The reflection component is described by the PEXRAV model (Magdziarz \& Zdziarski 1995), while for the iron line we used either a narrow Gaussian line or a relativistic line (DISKLINE model); in the latter model we kept fixed the inner radius at $6 r_{\mathrm{g}}$, leaving therefore as free parameters the outer radius and the inclination angle (the latter linked to that of the reflection component). We note that a broad Gaussian line, besides having no astrophysical meaning in the context under discussion, has the same number of free parameters as the relativistic lines, and therefore is not a simpler model. We therefore did not use this line parametrization in our fits. We tried to apply the relativistic effects to the reflection component too, adopting the REFSCH model, but no appreciable difference could be observed, due to the limited statistics.

This model (whatever the line parametrization) provides a good description of the data between 2 and $200 \mathrm{keV}$, but when extrapolated to lower energies (see Fig. 3) it falls significantly short of the data, suggesting the presence of a soft excess.

When the fit is performed over the whole band, this model gives an acceptable fit $\left(\chi^{2}=135.4 / 121\right.$ degrees of freedom (dof)) after steepening the power law index and simultaneously increasing the reflection component, but features in the residuals in the soft part of the spectrum are still present. We thus checked if the presence of 
Table 2. Best fit parameters for the two models described in Sect. 4.1 for Mrk 841 . The values followed by ${ }^{*}$ are kept fixed in the fit.

\begin{tabular}{|c|c||c|c|c|}
\hline & & PEXRAV+ABSORI model & PEXRAV+BB model & COMPT. +BB model \\
\hline \hline WABS & $N_{\mathrm{H}}$ & $2.34 \times 10^{20} \mathrm{~cm}^{-2 *}$ & $2.34 \times 10^{20} \mathrm{~cm}^{-2} *$ & $2.34 \times 10^{20} \mathrm{~cm}^{-2} *$ \\
\hline \hline PEXRAV & $\Gamma$ & $2.28_{-0.06}^{+0.04}$ & $2.12_{-0.02}^{+0.10}$ & - \\
\hline & $R$ & $4.6_{-1.4}^{+0.6}$ & $3.2_{-1.0}^{+1.6}$ & - \\
\hline & $\cos i$ & $0.80_{-0.53}^{+0.20}$ & $0.82_{-0.41}^{+0.09}$ & - \\
\hline & $E_{\mathrm{c}}$ & $>150 \mathrm{keV}$ & $>100 \mathrm{keV}$ & - \\
\hline \hline COMPT. & $T_{\text {soft }}$ & - & - & $71_{-8}^{+6} \mathrm{eV}$ \\
\hline & $\cos i$ & - & - & $0.8^{*}$ \\
\hline & $\Theta$ & - & - & $0.13_{-0.02}^{+0.01}$ \\
\hline & $\tau$ & - & - & $0.80_{-0.06}^{+0.13}$ \\
\hline & $R$ & - & - & $3.1_{-0.8}^{+1.6}$ \\
\hline \hline ABSORI & $\xi$ & $530_{-260}^{+1000} \mathrm{erg} \mathrm{cm} \mathrm{s}^{-1}$ & - & - \\
\hline & $N_{\mathrm{H}}$ & $\left(1.1_{-0.5}^{+1.6}\right) \times 10^{22} \mathrm{~cm}^{-2}$ & - & - \\
\hline \hline BB & $k T$ & - & $95_{-10}^{+10} \mathrm{eV}$ & $182_{-45}^{+56} \mathrm{eV}$ \\
\hline \hline ZDISK & $E$ & $6.4 \mathrm{keV}^{*}$ & $6.4 \mathrm{keV}$ & $6.4 \mathrm{keV}^{*}$ \\
\hline & $E W$ & $210_{-140}^{+120} \mathrm{eV}_{-0}^{+15} r_{\mathrm{g}}$ & $260_{-80}^{+120} \mathrm{eV}$ & $390_{-140}^{+150} \mathrm{eV}$ \\
\hline & $r_{\text {out }}$ & $6.01_{-3.0}^{+4.4} r_{\mathrm{g}}$ & $6.01_{-0}^{+4.8} r_{\mathrm{g}}$ \\
\hline \hline \hline$\chi^{2} /$ dof & $121.8 / 119$ & $120.8 / 119$ & $118.8 / 120$ \\
\hline \hline N.H.P. & 0.41 & 0.44 & 0.51 \\
\hline
\end{tabular}

a warm absorber is consistent with the data, by adding to the baseline model the ABSORI component. The addition of this component significantly improves the fit $\left(\Delta \chi^{2}\right.$ (dof) $=$ 13.6(2), corresponding to a probability of $99.8 \%$ according to the $\mathrm{F}$-test). The best fit parameters are listed in Table 2, first column. The value of $R, 4.6$, is very high, while the equivalent width of the iron line is $210 \mathrm{eV}$, inconsistent with the large value of $R$ found (e.g. Matt et al. 1992). There is also a problem with the iron line properties, as the value of $r_{\text {out }}$ is basically equal to $r_{\text {in }}$. If we use a simple, narrow Gaussian profile (ZGAUSS model) instead of the relativistic one for the iron line, we get a fit of similar quality $\left(\chi^{2}=123.9 / 120 \mathrm{dof}\right)$. The value for $R$ is also similar $\left(4.3_{-1.6}^{+9.1}\right)$, but the $E W$ is much lower $\left(80_{-60}^{+40} \mathrm{eV}\right)$, making more acute the inconsistency with the large value of $R$. In the following fits we will therefore consider only the relativistic line, keeping in mind that this choice is based on astrophysical plausibility rather than statistical arguments. Excluding altogether the line from the model, we found a still acceptable fit $\left(\chi^{2}=129.6 / 121\right.$ dof $)$, but again this is rather unplausible given the unambiguous presence of a reflection continuum (in the fit without the line the value of $R$ raises to 6.4!). According to the F-test, the presence of the (relativistic) line is significant at the 98.5\% confidence level.

Of course, excluding the reflection component leads to a very bad fit, as illustrated in Fig. 4, obtained by fitting the data with a simple power law instead of the PEXRAV model.

The next step was to fit the data with the baseline model plus a thermal emission (BB model), instead of the

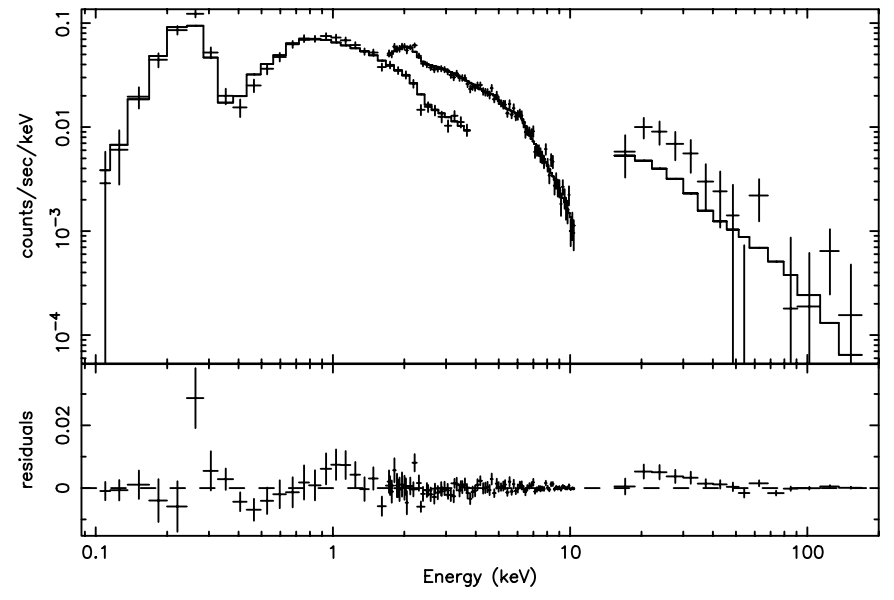

Fig. 4. BeppoSAX LECS+MECS+PDS data and best fit model for Mrk 841, when the reflection component is not included. An excess of counts in the PDS band is clearly present.

warm absorber (second column of Table 2). This results in a similar $\chi^{2}(120.8 / 119$ dof $)$. The equivalent width of the iron line is higher $\left(260_{-80}^{+120} \mathrm{eV}\right)$, thus becoming more consistent with the large value of the reflection component. Even if the value for $r_{\text {out }}$ is still very low, it is higher than before. Moreover, $R$ is lower and significantly better constrained than in the previous fit, being $3.2_{-1.0}^{+1.6}$. As in the previous fit, only a lower limit can be put on the high energy cut-off.

Since the value of the solid angle of the reflection component is still very high, we checked whether this could be entirely ascribed to an excess of counts in the PDS. 


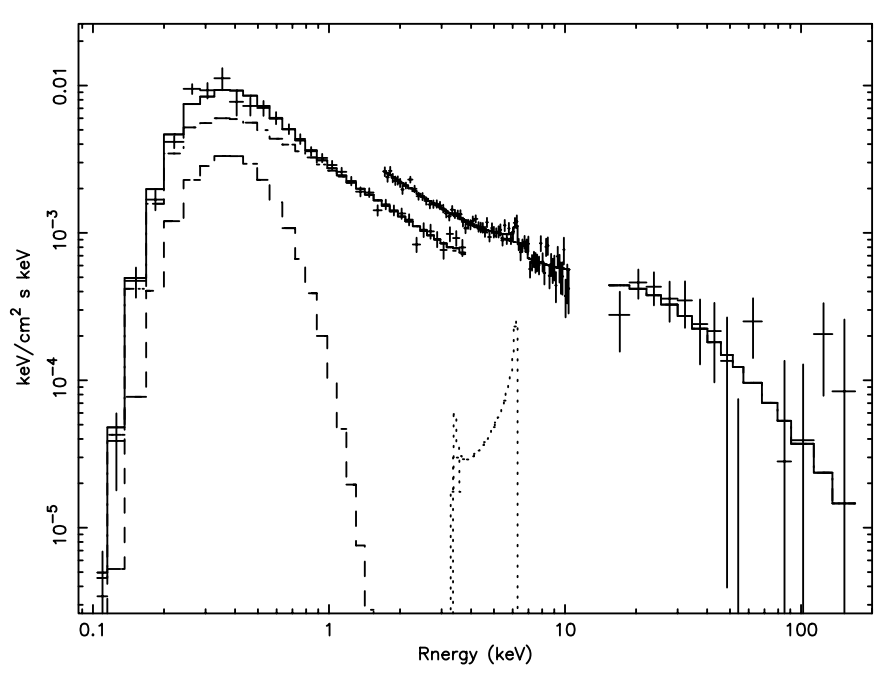

Fig. 5. BeppoSAX LECS+MECS+PDS unfolded spectrum and best fit model (second column of Table 2) for Mrk 841.

Thus we fitted the same model with LECS and MECS data only, without the PDS. The resulting $\chi^{2}$ is 108.9 /105 dof: the value of $R$ is still very high $(\simeq 3)$, therefore excluding that it may arise from a miscalibration between the MECS and the PDS instruments, or to contaminating sources in the PDS field of view.

We also tried to model the soft excess with a power law instead of a black body. The fit is significantly worse $\left(\chi^{2}=131.5 / 119\right.$ dof $)$, even adding a warm absorber $\left(\chi^{2}=125.8 / 117\right.$ dof $)$, but in the last case a lower value for $R(2.7)$ and a more consistent iron line $E W(250 \mathrm{eV})$ is found.

On the contrary, the fit with the bremsstrahlung emission provides a fit almost as good $\left(\chi^{2}=123.4 / 119\right.$ dof $)$ as with the black body. The value of $R, 2.3$, is lower and consistent, within the errors, with the value of the iron line $E W, 280 \mathrm{eV}$.

We next tried to fit the primary continuum with a more physical model than a simple power law; we used the Comptonization model of Haardt \& Maraschi (1993) (third column of Table 2). The corresponding XSPEC code is in the form of a grid of spectra; the fitting parameters are the electron temperature in units of $m c^{2}$, $\Theta$, the optical depth $\tau$, and the temperature of the soft black body emission, $T_{\text {soft }}$; the parameters range from 0.1 to $1(\Theta), 0.05$ to $1(\tau)$ and 5 to $100 \mathrm{eV}(\Theta)$. The cosine of the inclination angle has been fixed to 0.8 (as suggested by the PEXRAV plus DISKLINE fit), in order to reduce the number of free parameters. The Compton reflection component is also included in the model. The fit is satisfactory $\left(\chi^{2}=118.8 / 120\right.$ dof $)$, but the problem of the large value of $R$ is not solved. The line $E W$ is about $390 \mathrm{eV}$ (obtained with the diskline model). The parameters of the continuum are $\tau=0.8$ and $\Theta=0.13$. It is worth noting that the soft emission present in the Comptonization model is not sufficient, and a further black body component is also required, suggesting either a multicolor disc emission or an altogether different component.
If a bremsstrahlung is used instead of a black body, the fit is significantly worse $\left(\chi^{2}=135.0 / 120\right.$ dof $)$. A power law, instead, provides a fit of comparable quality $\left(\chi^{2}=\right.$ $121.0 / 120$ dof), but the problem of the large value of $R$ is made even more serious. Finally, a warm absorber, instead of a true soft excess, gives a significantly worse fit $\left(\chi^{2}=\right.$ 138.6/120 dof).

\subsection{Mrk 335}

We firstly proceeded as for Mrk 841, and fitted the data above $2 \mathrm{keV}$ with the same baseline model, with a Galactic column density $N_{\mathrm{H}}=4.0 \times 10^{20} \mathrm{~cm}^{-2}$. Given the poor statistics in the PDS, we did not include the high energy cut-off. The extrapolation to lower energies is shown in Fig. 6: the soft excess is even more clear than for Mrk 841. In fact, fitting this model to the whole band, we obtain a totally unacceptable fit, with $\chi^{2}=286.4 / 128$ dof (see Fig. 7). The inclusion of a warm absorber strongly improves the fit $\left(\chi^{2}=151.4 / 126\right.$ dof $)$, but still a power law is needed to model the soft excess $\left(\chi^{2}=134.2 / 124 \mathrm{dof}\right)$. The solid angle of the reflection component is rather low $(\sim 0.6)$ and ill constrained, while the equivalent width of the iron line is rather high, being $\sim 300 \mathrm{eV}$. If a narrow Gaussian line is used instead of the relativistic one, the fit is worse $\left(\chi^{2}=139.3 / 125\right.$ dof $)$.

A significant improvement in the fit $\left(\Delta \chi^{2} \simeq 8.2\right.$, corresponding to a probability of about $99.4 \%$ according to the $\mathrm{F}$-test) is obtained adding a narrow Gaussian line to the relativistic one. The new model (see the first column of Table 3) has parameters not very different from the previous one, but the total equivalent width of the iron line is very large, the sum of the two components being $270+390=660 \mathrm{eV}$.

If a black body or a bremsstrahlung is used instead of the power law, rather worse fits are found $\left(\chi^{2}=\right.$ $130.7 / 123$ dof for the black body, $\chi^{2}=133.2 / 123$ dof for the bremsstrahlung).

Recently, Ballantyne et al. (2001) have found that a good fit to the ASCA spectrum of Mrk 335 is achieved with the ionized disc model (Ross \& Fabian 1993). This could naturally explain the large equivalent width of the iron line (Matt et al. 1993, 1996). We therefore substituted the model PEXRIV, which allows for ionization of the reflecting matter, to PEXRAV, which is for neutral matter alone. We also fixed the energy of the iron line in the DISKLINE model either to 6.7 (He-like iron) or to 6.97 ( $\mathrm{H}$-like iron), and excluded from the fit the narrow line. The results are inconclusive: the $\chi^{2}$ is not improved (126.1/123 dof for the H-like iron, and 126.8/123 dof for the He-like iron), and the reflection component in both cases goes to zero (but remaining ill-constrained). The equivalent width of the iron line is $690 \mathrm{eV}$ for the $\mathrm{H}$-like iron, and $660 \mathrm{eV}$ for the He-like iron. Very similar results are obtained using the Ross \& Fabian $(1993)^{1}$ model.

\footnotetext{
1 Available at http://heasarc.gsfc.nasa.gov/docs/xanadu/ xspec/models/iondisc.html
} 


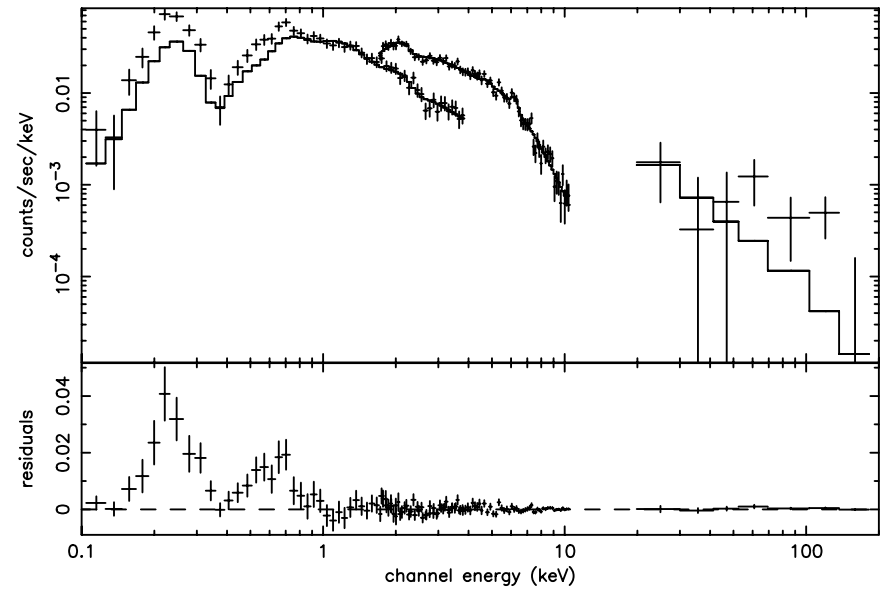

Fig. 6. BeppoSAX LECS+MECS+PDS data and best fit model above $2 \mathrm{keV}$ for Mrk 335, then extrapolated to lower energies to illustrate the presence of a soft excess.

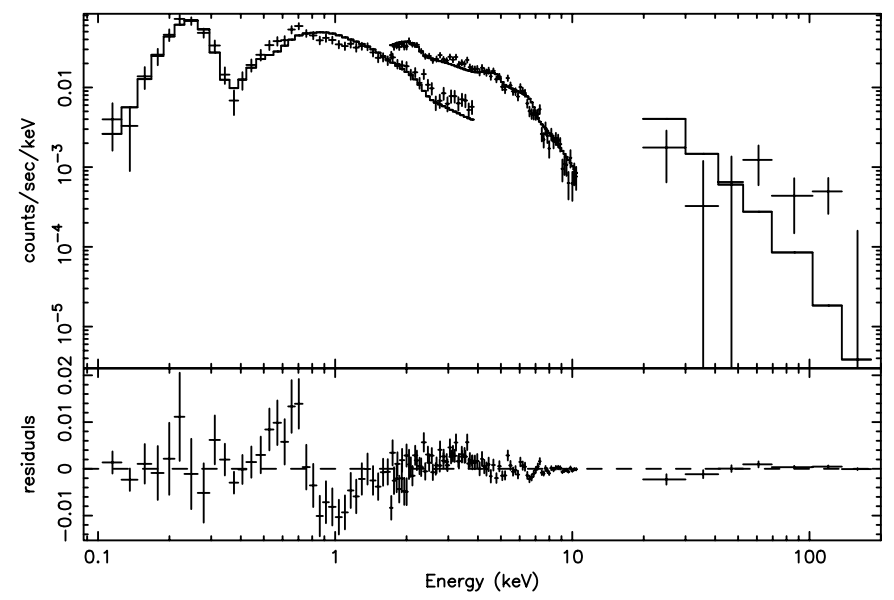

Fig. 7. Data and best fit model for Mrk 335, when the baseline model is adopted. The fit is clearly inadequate below $\sim 2 \mathrm{keV}$, due both to the warm absorber and a soft excess (see text for details).

We therefore conclude that the ionized disc model is neither proved nor disproved by the data, remaining the more plausible on physical ground.

We then fitted the spectrum with the Comptonization model described in the previous section, using a power law, a black body or a bremsstrahlung model for the soft excess. In all cases, the fit is slightly better than that with a cut-offed power law. The best fit is found for a black body (121.4/123 dof, second column of Table 3), while for the power law and the bremsstrahlung we found a reduced $\chi^{2}$ of 124.5/123 dof and 123.2/123 dof, respectively. None of these fits require a narrow iron line besides the relativistic one.

As far as the reprocessing components are concerned, we still found a very large iron line, and a Compton reflection component consistent with zero.

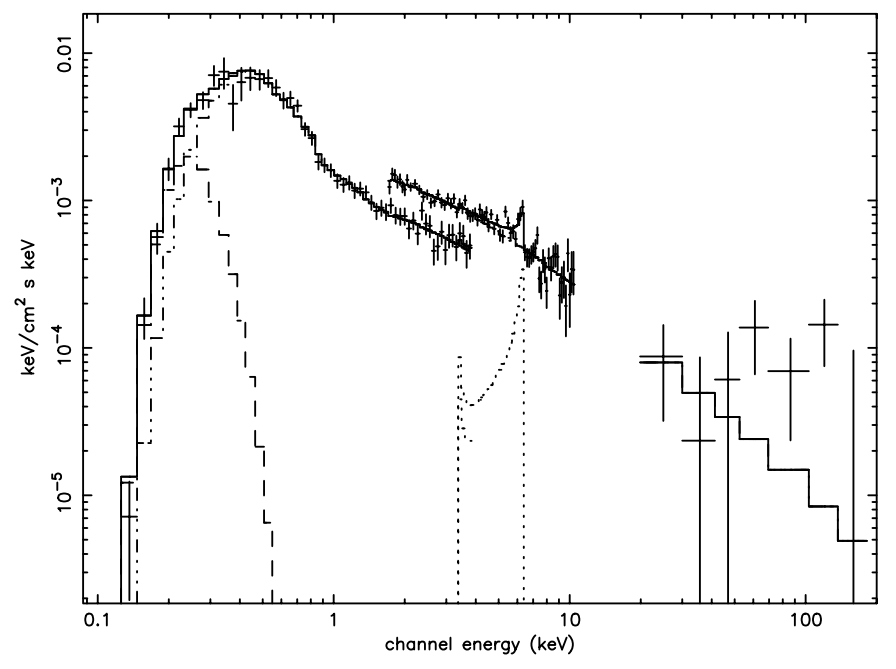

Fig. 8. BeppoSAX LECS+MECS+PDS unfolded spectrum and best fit model (second column of Table 3) for Mrk 335.

\section{Discussion and conclusions}

We analysed the BeppoSAX data of Mrk 841 and Mrk 335, two Seyfert 1s in which previous observations indicated the presence of a soft excess. We indeed confirm the presence of this component, even if for Mrk 841 the fit with the warm absorber is almost as good as that with a true soft excess. The quality of the data are not, unfortunately, good enough to choose between different parameterizations of the soft emission. In fact, for Mrk 841 we found that a power law is a significantly poorer description of the soft excess, but a black body and a bremsstrahlung are equally acceptable (even if the former is more plausible if the soft excess is indeed associated with the accretion disc), at least as long as a power law is used for the hard X-ray component. For Mrk 335, the presence of the soft excess is clear. If a simple power law is used for the hard component, then a power law is also to be preferred for the soft excess. If, instead, the hard X-ray emission is described by the Comptonization model of Haardt \& Maraschi (1993), the black body and the bremsstrahlung provide a better fit. To quantify the relative importance of the soft excess, in Table 4 the bolometric and 0.1$0.5 \mathrm{keV}$ fluxes in the black body component, for both the PEXRAV and Comptonization models, are given for both sources. Comparing them with the $2-10 \mathrm{keV}$ fluxes given in Table 1, it is clear that the soft excess is more important in Mrk 335, not surprisingly as it is a NLS1. Rather unexpectedly, for Mrk 335 the largest fluxes are obtained in the Comptonization model, despite the presence of a further thermal component in the model.

Comparing our results with previous observations, we note that the soft excess in Mrk 841 is significantly weaker than that found by Arnaud et al. (1985), who however found a much flatter spectral index $(\Gamma=1.6)$ for the hard component. Because at that time the spectral complexities of Seyfert 1s have not yet been discovered, it is possible that they overestimated the soft excess (see the 
Table 3. Best fit parameters for the two models described in Sect. 4.2 for Mrk 335. The values followed by ${ }^{*}$ are kept fixed in the fit.

\begin{tabular}{|c|c|c|c|}
\hline & & PEXRAV+POW & СомРт.+BB \\
\hline WABS & $N_{\mathrm{H}}$ & $4.0 \times 10^{20} \mathrm{~cm}^{-2 *}$ & $4.0 \times 10^{20} \mathrm{~cm}^{-2 *}$ \\
\hline \multirow[t]{4}{*}{ PEXRAV } & $\bar{\Gamma}$ & $2.04_{-0.03}^{+0.17}$ & - \\
\hline & $R$ & $0.58_{-0.58}^{+1.47}$ & - \\
\hline & $\cos i$ & $0.90_{-0.20}^{+0.05}$ & - \\
\hline & $E_{\mathrm{c}}$ & $\infty^{*}$ & - \\
\hline \multirow[t]{5}{*}{ COMPT. } & $T_{\text {soft }}$ & - & $98_{-4}^{+2} \mathrm{eV}$ \\
\hline & $\cos i$ & - & $0.8^{*}$ \\
\hline & $\Theta$ & - & $0.14_{-0.03}^{+0.03}$ \\
\hline & $\tau$ & - & $0.62_{-0.09}^{+0.16}$ \\
\hline & $R$ & - & $0_{-0}^{+1.6}$ \\
\hline \multirow[t]{2}{*}{ ABSORI } & $\overline{\xi \xi}$ & $510_{-40}^{+60} \mathrm{erg} \mathrm{cm} \mathrm{s}^{-1}$ & $890_{-740}^{+1060} \mathrm{erg} \mathrm{cm} \mathrm{s}^{-1}$ \\
\hline & $N_{\mathrm{H}}$ & $\left(4.2_{-0.3}^{+0.7}\right) \times 10^{22} \mathrm{~cm}^{-2}$ & $\left(2.1_{-1.7}^{+2.2}\right) \times 10^{22} \mathrm{~cm}^{-2}$ \\
\hline POWERLAW & 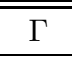 & $2.68_{-0.04}^{+0.08}$ & - \\
\hline $\mathrm{BB}$ & $k T$ & - & $29_{-7}^{+7} \mathrm{eV}$ \\
\hline \multirow[t]{2}{*}{ ZGAUSS } & $E$ & $6.4 \mathrm{keV}^{*}$ & - \\
\hline & $E W$ & $270_{-70}^{+65} \mathrm{eV}$ & - \\
\hline \multirow[t]{3}{*}{ ZDISKLINE } & $E$ & $6.4 \mathrm{keV}^{*}$ & $6.4 \mathrm{keV}^{*}$ \\
\hline & $r_{\text {out }}$ & $6.01_{-0}^{+3.3} r_{\mathrm{g}}$ & $6.02_{-0.01}^{+1.62} r_{\mathrm{g}}$ \\
\hline & $E W$ & $390_{-89}^{+86} \mathrm{eV}$ & $760_{-220}^{+230} \mathrm{eV}$ \\
\hline \multicolumn{2}{|c|}{$\overline{\chi^{2} / \text { dof }}$} & $12126.0 / 123$ & $121.4 / 123$ \\
\hline \multicolumn{2}{|c|}{ N.H.P. } & 0.41 & 0.53 \\
\hline
\end{tabular}

Introduction). Our results are instead remarkably similar to those found by Nandra et al. (1995) analysing ROSAT data (compare model B of their Table 1 with the second column of our Table 2 and with Table 4 ).

As far as the soft excess in Mrk 335 is concerned, comparing our results with those of Reynolds (1997, see his Table 5), based on ASCA data, we note that the black body bolometric luminosity in our observation is a factor 2.7 higher than in the ASCA observation, while the 2-10 keV luminosities are very similar (with the photon indices being also very similar). This finding rules out models in which the soft excess is entirely due to reprocessing of the hard X-ray component, because in such a model the two components should vary together.

The Comptonization model gives a slightly better fit for Mrk 335, and a comparable one for Mrk 841. While the quality of the spectra, especially at high energies, is not good enough to allow definitive conclusions, it may be instructive to compare the present results with those obtained, adopting the very same model, by Petrucci et al. (2001, P01) on a sample of Seyfert 1s observed by BeppoSAX. For both sources the best fit value for the temperature of the plasma is about $70 \mathrm{keV}$, significantly lower than any value in the P01 sample. Looking at their Fig. 2, we can see that the values we find of $\Theta$ and $\tau$ are in between the theoretical curves for a plane-parallel ("slab") geometry and for an hemispherical one (in this
Table 4. Fluxes and luminosities in the black body component.

\begin{tabular}{|c|c|c|c|c|}
\hline \multirow[b]{2}{*}{ Source } & \multicolumn{2}{|c|}{$\begin{array}{c}\text { PEXRAV+BB } \\
\text { Flux } /[\text { Lum. }] \\
\left(10^{-11} \mathrm{erg} \mathrm{cm}^{-2} \mathrm{~s}^{-1}\right) \\
{\left[\left(10^{43} \mathrm{erg} \mathrm{s}^{-1}\right)\right]}\end{array}$} & \multicolumn{2}{|c|}{$\begin{array}{c}\text { Compt+BB } \\
\text { Flux } /[\text { Lum. }] \\
\left(10^{-11} \mathrm{erg} \mathrm{cm}^{-2} \mathrm{~s}^{-1}\right) \\
{\left[\left(10^{43} \mathrm{erg} \mathrm{s}^{-1}\right)\right]}\end{array}$} \\
\hline & Bol. & $0.1-0.5 \mathrm{keV}$ & Bol. & $0.1-0.5 \mathrm{keV}$ \\
\hline Mrk 841 & $\begin{array}{l}0.69 \\
{[3.9]}\end{array}$ & $\begin{array}{l}0.51 \\
{[2.9]}\end{array}$ & $\begin{array}{l}0.36 \\
{[2.0]}\end{array}$ & $\begin{array}{l}0.12 \\
{[0.7]}\end{array}$ \\
\hline Mrk 335 & $\begin{array}{c}1.2 \\
{[3.5]}\end{array}$ & $\begin{array}{c}1.0 \\
{[2.9]}\end{array}$ & $\begin{array}{c}5.6 \\
{[16.1]}\end{array}$ & $\begin{array}{c}2.9 \\
{[10.9]}\end{array}$ \\
\hline
\end{tabular}

respect, in agreement with most of the sources analyzed by P01; note also that, for Mrk 335, a pure plane-parallel geometry is ruled out by the comparison of BeppoSAX and ASCA results, see above). The need for a further black body component may suggest that the correct geometry is the patchy one, as envisaged by Haardt et al. (1994), at least for Mrk 841. In the case of Mrk 335, instead, the fact that the temperature of the extra black body is greater than that of the Comptonized one makes this solution hardly tenable.

For both sources the most puzzling results concern the reprocessed components. In Mrk 841 we found a very large value for the solid angle, $R$, of the Compton reflection 
component, while the $E W$ of the iron line, supposed to originate in a relativistic disc, is larger than usual but not so extreme, at least as far as the face values are considered. Actually, within the errors a solution in which both components are about a factor 2 larger than in the $\Omega=2 \pi$ case is possible (in agreement with previous GINGA observations, in which very large values for both the iron line $E W$ and the amount of reflection continuum were found, George et al. 1993). This may be explained either by an anisotropy of the illuminating radiation (see e.g. Ghisellini et al. 1991, where it is shown that even larger anisotropies may be achieved), or by a delayed response of the reprocessing components to variations of the primary continuum (but the latter possibility implies a distance of the reprocessing matter greater than that of the inner accretion disc, in disagreement with the hypothesis of a relativistic iron line necessary to have a sufficent $E W$ to match the large amount of reflection). Interestingly, the large value of $R$ seems to follow the anticorrelation between $R$ and $\Theta$ found by P01, as well as that the correlation between $R$ and $\Gamma$ found by Zdziarski et al. (1999).

In Mrk 335, on the contrary, we found a very large iron line (in agreement with previous findings) but small or moderate Compton reflection component (the upper limit being between 1 and 2, depending on the model for the continuum). A possible explanation could be a largerthan-solar iron abundance, but the values required are rather high, i.e. around 10 (Matt et al. 1997). A more viable solution is in terms of an ionized disc; if the iron is mainly in $\mathrm{He}-$ and $\mathrm{H}$-like ions, an equivalent width several times that for neutral iron can be obtained, at least in the constant density solution (Ross \& Fabian 1993; Matt et al. 1993, 1996). It is worth noting that Mrk 335 is usually classified as a Narrow Line Seyfert 1 galaxy, a class of sources in which significant ionization of the accretion disc is often observed (see e.g. Comastri 2000 and references therein), not surprisingly as these sources are commonly believed to accrete at high rates.

Acknowledgements. The BeppoSAX satellite is a joint ItalianDutch program. We thank the BeppoSAX Scientific Data Centre for assistance, M. Guainazzi for helpful discussions, and the anonymous referee for comments that helped improving the clarity of the paper. This research has made use of the NASA/IPAC Extragalactic Database (NED) which is operated by the Jet Propulsion Laboratory, California Institute of Technology, under contract with the National Aeronautics and Space Administration. This work was supported by the Italian Space Agency, and by the Ministry for University and Research (MURST) under grants COFIN98-02-32 and COFIN0002-36.

\section{References}

Arnaud, K. A., Branduardi-Raymont, G., Culhane, J. L., et al. 1985, MNRAS, 217, 105

Ballantyne, D. R., Iwasawa, K., \& Fabian, A. C. 2001, MNRAS, in press

Boella, G., Butler, R. C., Perola, G. C., et al. 1997, A\&AS, 122, 299

Comastri, A. 2000, New As. Rev., 44, 403

Fiore, F., Elvis, M., McDowell, J. C., Siemiginowska, A., \& Wilkes, B. J. 1994, ApJ, 431, 515

Ghisellini, G., George, I. M., Fabian, A. C., \& Done, C. 1991, MNRAS, 248, 14

George, I. M., Nandra, K., Fabian, A. C., et al. 1993, MNRAS, 260,111

George, I. M., Turner, T. J., Netzer, H., et al. 1998, ApJS, 114,73

Guainazzi, M., Nicastro, F., Fiore, F., et al. 1998, MNRAS, 301, L1

Haardt, F., \& Maraschi, L. 1993, ApJ, 413, 507

Haardt, F., Ghisellini, G., \& Maraschi, L. 1994, ApJ, 432, L95

Iwasawa, K., Fabian, A. C., \& Nandra, K. 1999, MNRAS, 307, 611

Magdziarz, P., \& Zdziarski, A. A. 1995, MNRAS, 273, 837

Matt, G. 2000, Astron. Lett. Comm., in press

Matt, G., Perola, G. C., Piro, L., \& Stella, L. 1992, A\&A, 263, 453

Matt, G., Fabian, A. C., \& Ross, R. R. 1993, MNRAS, 262, 179

Matt, G., Fabian, A. C., \& Ross, R. R. 1996, MNRAS, 280, 823

Matt, G., Fabian, A. C., \& Reynolds, C. S. 1997, MNRAS, 289, 175

Mineo, T., Fiore, F., Laor, A., et al. 2000, A\&A, 359, 471

Nandra, K., Turner, T. J., George, I. M., et al. 1995, MNRAS, 273,85

Orr, A. 2000, New Astron. Rev., 44, 487

Petrucci, P. O., Haardt, F., Maraschi, L., et al. 2001, ApJ, submitted (PO1)

Piro, L., Matt, G., \& Ricci, R. 1997, A\&AS, 126, 525

Reynolds, C. S. 1997, MNRAS, 286, 513

Ross, R. R., \& Fabian, A. C. 1993, MNRAS, 261, 74

Turner, T. J., \& Pounds, K. A. 1989, MNRAS, 240, 833

Turner, T. J., George, I. M., \& Mushotzky, R. F. 1993b, ApJ, 412, 72

Turner, T. J., Nandra, K., Zdziarski, A. A., et al. 1993a, ApJ, 407, 556

Walter, R., \& Fink, H. H. 1993, A\&A, 274, 105

Walter, R., Orr, A., Courvoisier, T. J.-L., et al. 1994, A\&A, 285, 119

Wang, T., Brinkmann, W., \& Bergeron, J. 1996, A\&A, 309, 81

Zdziarski, A. A., Lubinski, P., \& Smith, D. A. 1999, MNRAS, 303, L11 